\title{
An Image Processing Based Novel Approach to Real-Time Analysis and Diagnosis of Epileptic Seizures using Wearable Sensors
}

\author{
Chandrashekhar G.Patil \\ Department of Electronics and Communication Engineering \\ Sinhgad Academy of Engineering, Pune, Maharashtra, India
}

\begin{abstract}
Electroencephalogram (EEG) is the traditional method to record the Epileptic seizures. Different Signal processing methods are used for analysis and diagnosis of these seizures. The treatment/therapy schedule and pattern are completely depending on precise recognition and quantification of types of seizures. Because of complexity and cost, the traditional method may be replaced by a new real-time system which is proposed here. Generally, the changes in facial expressions are observed during an Absence Epileptic seizures. Urine discharge is the major symptom observed in Tonicclonic type of Epileptic seizures. The combination of an Image processing based system and an embedded system (with wearable sensors) may help in efficient detection of absence seizures and Tonic-clonic seizures. The proposed system combines these two methods to get real-time detection of these Epileptic Seizures. Results show significant improvement ( $\mathbf{9 0 \%}$ ) in the rate of correct detection of seizure types.
\end{abstract}

Keywords - Image processing, Wearable sensors, Embedded system

\section{INTRODUCTION}

The EEG signals provide complex information about normal brain activities. Epileptic seizures represent disorganized neural activities in the brain [1]. The WHO (World Health Organization) has recently figured out that, nearly 50 Million people are suffering from Epilepsy worldwide. The major symptoms of this disease are repetitive seizures. During seizures, an abnormal movements of the human body or its parts along with unconsciousness and loss of control of bladder are generally noticed. To describe epilepsy properly, minimum two seizures are necessary. The Generalized seizures is an important class of Epileptic seizures with Absence seizures and tonic-clonic seizures as subclasses.

Recently some antiepileptic drugs have been introduced. Still many of people are facing problems [2]. The unpredictable nature of seizures creates serious consequences [3]. The improvement in efficiency of detection systems enhances therapies and may lead towards early detection of seizures. The great advantage of such system is that it tries to avoid accidents and minimizes injuries.

Different methods, techniques have been tested successfully for detection of seizures. Artificial Neural Networks (ANN) [4,5,6], Wavelet transforms [7,8], Support Vector Machines [9,10] etc. are few of the examples of such methods. Mathematical models of such algorithms consist of two major processes viz. feature extraction followed by classification process. The physiological data measured, is used for deriving appropriate features. These features are used to decide the presence or absence of seizure by application of proper thresholding or filtering process. An effect of Electrocardiogram (ECG) signals on epileptic seizures are studied and presented in [11]. Leutmezer F., and Opherk C. have shown the relationship between epileptic seizures and ECG signals. $[12,13]$. Besides this seizure detection, using motion sensors like Accelerometers, have also been proposed and tested [14,15]. Video detection system was also suggested using motion trajectory methods [16]. Wearable objects with some marking schemes are used for video analysis to record seizure activity in [17].

Each of these systems has merits and demerits. Most important demerits of this system are its sensitivity and specificity. Therefore for further improvement in these parameters, the combination of different methods are also suggested. EEG along with ECG analysis was proposed in [18], whereas an Accelerometer in addition to Support Vector Machine algorithm was suggested by Poh in 2012[19]. This literature survey leads us towards the conclusion that, algorithms based on Image Processing along with few of the wearable sensor may fulfill the requirements of the system. A real-time system that has the combination of Image processing based seizure detection method and sensors based embedded system is proposed in this paper. 
The rest of the paper is organized as follows. Section II explains the methodology of proposed algorithms. Experimental results are presented in section III. Concluding remarks are given in section IV.

\section{PROPOSED ALGORITHM}

\section{A. Seizure Detection algorithm -}

Absence seizure: An indication of simple absence seizure is a vacant stare, which may be mistaken for a lapse in attention that lasts 10 to 15 seconds, without any subsequent confusion, headache or drowsiness. Abrupt and abnormal changes in the movement, Lip smacking, Fluttering of eyelids, rubbing of fingers are few of the symptoms of absence seizures. The duration of Absence seizures generally is very less ranging from ten to fifteen seconds. The subject even could not even memorize this small episode of seizures. Repetitive episodes of this seizures may disturb day to day life of the subject.

Generalized tonic-clonic seizure: Here in this seizure, entire body may experience the seizure effect. It is also called grand mal seizure. Seizures, convulsion or epilepsy are most commonly used terms with respect to generalized tonic-clonic seizures. An aura is another medical term that relates the symptoms like changes in vision, taste, smell, or sensory systems; hallucinations, or dizziness experienced by many subjects before the occurrence of the seizure. The rigidness observed in muscular movements is the another major characteristics of this seizures. Sometimes this rigidness of muscles turned into violent activities of a subject or leads him towards unconsciousness. Besides this some of the other signs like biting the cheek or tongue, clenched teeth or jaw, discharge of urine or stool, obstacles in breathing, etc. are also observed during this seizures.

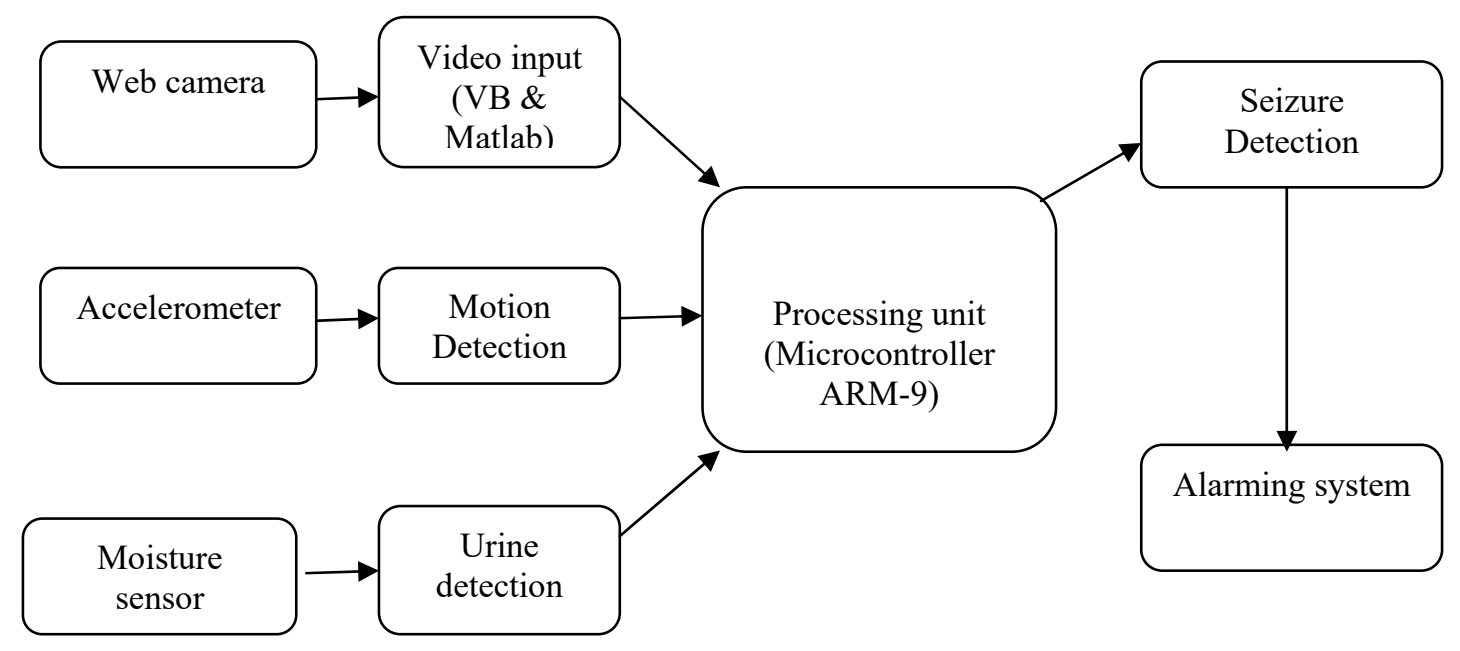

Figure 1. The Block diagram of the proposed system

The Image processing algorithms are proposed here to capture facial muscular movements occurred during this seizure. The facial nerve, known as motor nerve, controls muscles that move the eyebrows, close and widens the eyes, and move the mouth and lips. A cap, which has a video camera continuously focusing on the face of the patient, captures these movements and provide us the real-time information about the ongoing seizures. The similar type of algorithms has been undertaken for the study of Automatic human emotion recognition system[20]. Different emotions such as disgust, fear, joy etc. are recognized by these type of systems. The muscular movement like tightening of the lips, lowering the lip corners, etc. represents various moods and emotions in sadness.

A human-observer-based coding system [21] encodes movements and emotions. Pediaditis et. al. [22] reviewed vision based human motion analysis from Epileptic seizure detection point of view. An image processing techniques have been applied using quantitative video analysis of movement patterns of adult patients with epilepsy during seizures [23].The suitability of wearable sensors (accelerometer) is used to detect seizure events in patients exhibiting different types of seizures has been evaluated by Nijsen et. al. [14]. The block diagram of the proposed system is as shown in Fig.1.

As shown in the block diagram there are two types of inputs to the processor. First is the video input and the second one consists of inputs from wearable sensors viz. 1) Accelerometer and 2) Moisture sensor. The motion sensor i.e. 
Accelerometer detects the abnormal and repetitive type of the motion of the head of the patient under observation. The popular Viola-Jones detection algorithm followed by a correlation function is proposed here for detection of faces and region of interest in the face.

B. Region of Interest detection -

Widely used Viola-Jones algorithm is used here to detect the region of interest from the face of the patient. The region of interest is decided by keen observation of an epileptic patient with ongoing seizures. Mouth region and eye region are found to be the regions of interest where various movements of the muscles have been observed during the seizures. For this, database that consists of 789 videos of @ 300 seconds each with the recording of epileptic seizures especially absence and tonic-clonic seizures have been used for extraction of features. The flow chart for the detection of types of seizures is as shown in Fig. 2

Haar-like features are used to detect a face in this algorithm. Papageorgiou et al. [24] have suggested these simple features. Here different windows or rectangles are defined to specify the features. A number of features may be generated using these Haar-like features. It depends on the number of rectangles used. For example, if two rectangles are used then it is the two-rectangle feature, if three rectangles are used then it is a three-rectangle feature and so on. The difference between the sum of the pixels within two rectangular regions represents a value of a tworectangle feature. A three-rectangle feature computes the sum within two outside rectangles subtracted from the sum in a center rectangle. Finally, a four-rectangle feature computes the difference between diagonal pairs of rectangles [25].For fast computation of these rectangle features, an intermediate representation of the image integral image is used. At any location (x, y), an integral image represents an image that consists of the sum of the pixels above and to the left of $(x, y)$.

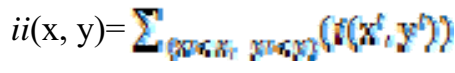

where $i i(\mathrm{x}, \mathrm{y})$ is an integral image and $i(\mathrm{x}, \mathrm{y})$ is the original image as shown in Figure 3

The box car image ' $r$ ' is calculated with the help of these integral images as shown below

i . $r=\left(\iiint_{i}, r^{\prime \prime m}\right.$

This box car image consists of the pixels whose sum is given by different four arrays calculated by considering various integral images as shown in Figure 3. The resolution of this window is $24 \times 24$. For the image with size [256,256], a number of boxcar images would be about 1,60,000 (by considering different permutation and a combination of rectangle features with different sizes).

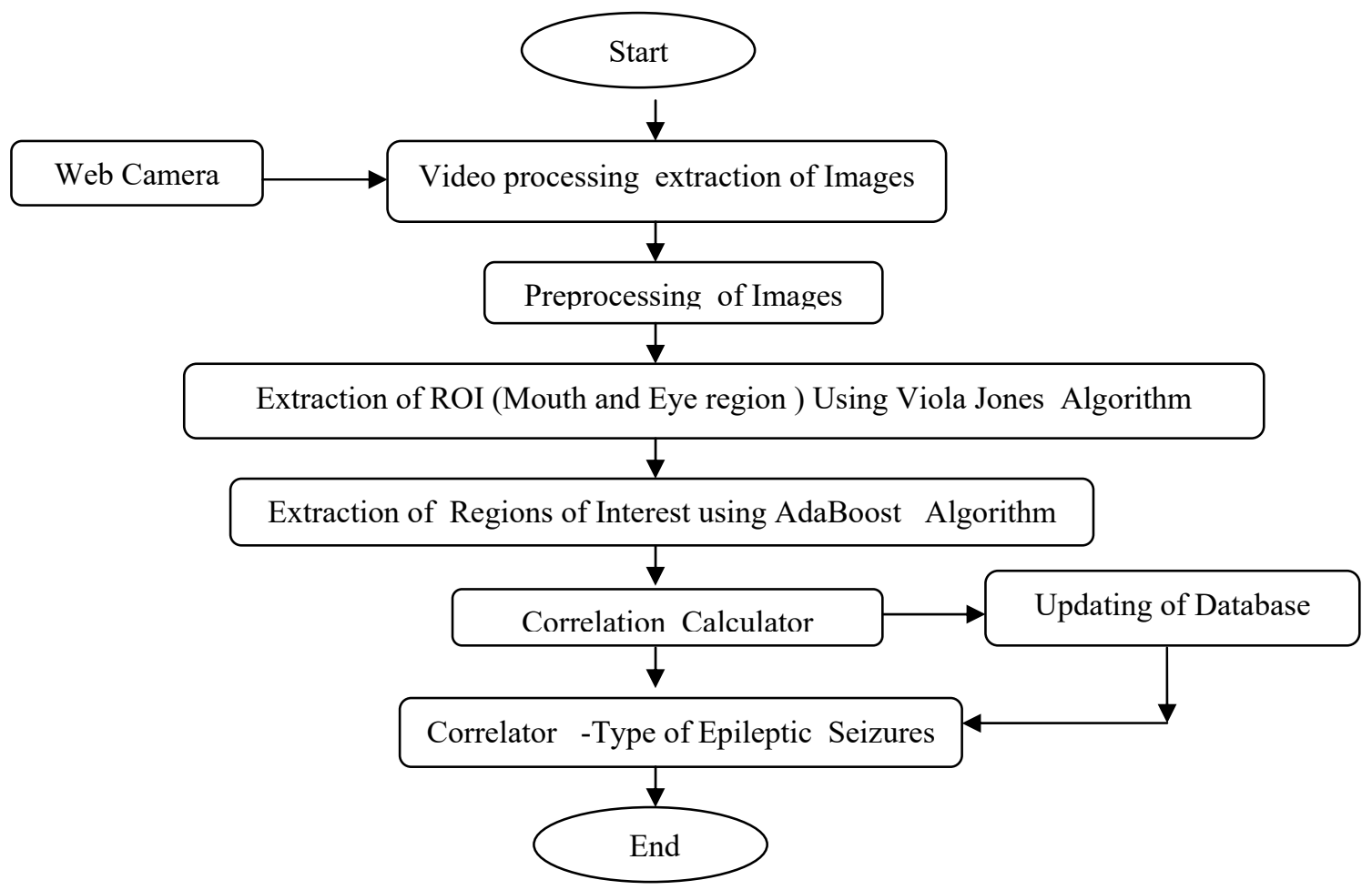


Figure 2 Flow chart of Detection of type of Epileptic Seizure

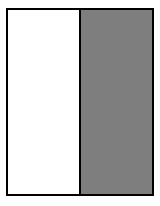

(A)

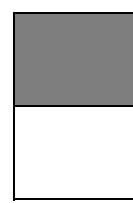

(B)

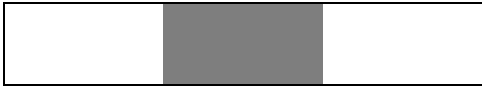

(C)

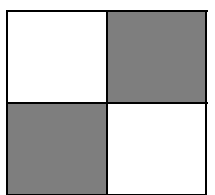

(D)

Figure 3. A) \& B) Two rectangle window C) Three rectangle window D) Four rectangle window

The proper setting of the region of interest is necessary. To detect exact regions of our interest, to have smaller overhead and rule out most false positives The target size (face) window is moved over the input image. The haar-like feature is calculated for every subsection of the image. This calculated difference value is then compared to a learned threshold which separates non-objects from objects. To describe an object with sufficient accuracy a large number of Haar-like features are necessary.

Similar to the face detection, Haar-like features are used to locate the mouth. Canny edge detection and masks are used to detect the corners of the mouth. Two different masks which represent the corners of the mouth are considered. These masks are moved from top to bottom to calculate how many positive bits are found within the specific region. A similar method is again used to find the upper and lower lip. Here corners of the mouth are used as starting and ending point for masks of a lip. To determine the position of the eyes the same algorithm is used but with a different haar classifier in the appropriate regions of interest. These regions are the upper left and upper right part of the known face region as shown in Fig.4.

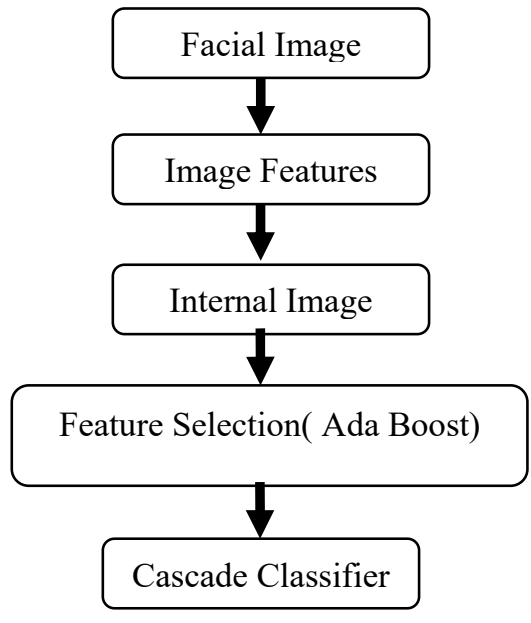

Figure 5 Viola-Jones Detection Flow

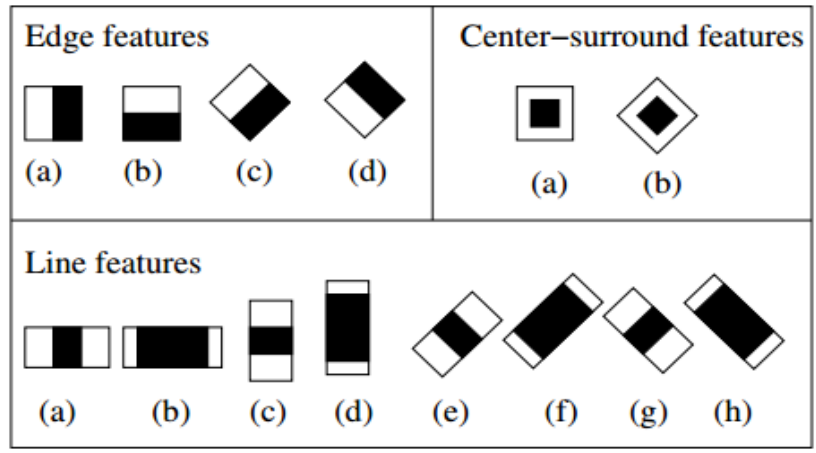

Figure 4 Used Feature Prototypes

For Eyebrow detection, Canny edge detection method is used. Canny edge detection is applied on the ROI above the eye. The mask slides top to bottom to find out the most edges within its shape. Based on the position of the eyes an angle is calculated to draw a mask. A running average is used to minimize false positives. After extracting the facial features of face, eye, mouth and eyebrows a very vital step is done by correlation function. Correlation function compares the features of live taken image to that of the data base. Detection of epilepsy depends on the correlation value which is a threshold value. It is a widely used method for object detection. Training is slow, but detection is very fast. Viola-Jones algorithm flows according to Figure 5.

After calculating facial features, each feature is compared with stored database with correlation coefficient.

$$
r=\operatorname{Sum}\left(Z x^{*} Z y\right) /(n)
$$


where, $r=$ Correlation coefficient $\quad n=$ No. of scores

The threshold value (correlation coefficient) is set as 0.3 discriminating normal behavior and epilepsy. As per the patient, the threshold value can be changed.

Wearable sensors: To support this automatic epilepsy seizure detection system, two sensors are used to sense 1.motion of the head of the subject under observation and 2.moisture sensor to sense the urine discharge. Most of the epileptic seizures are observed with these symptoms. Therefore this sensor information will assist the system to ensure the epileptic seizures

Accelerometer: A 2-dimensional capacitive sensing accelerometer is used here to sense the motion of the head of the subject. Here accelerometer provides an output voltage depending on the distance between two planar surfaces. The change in distance between these plates during the motion of the head deviates capacity from its null point. The corresponding output voltage is applied to the central processor. Because of the high accuracy and sensitivity of this sensor, the system becomes stable and reliable. Low noise immunity, power dissipation, and large bandwidth are few of the advantages of this sensor.

Moisture Sensor: Discharge of urine during the tonic-clonic seizure is another major symptom of epilepsy. The output of this sensor is used to ensure the seizure. It can be wear along with the clothes. A threshold is required to differentiate between moisture due to the discharge of urine and moisture due to atmospheric conditions.

Indicators: The system is open loop system. It only monitors the change in predefined conditions of the subject and alerts the expert system. Here both audio and video type of indicators are used to alert the corrective system.

\section{Experiment and Result}

The experiments carried out in one of the renown hospitals and the results are validated by Medical experts. Total 100 subjects suffering from epilepsy were under observation for 15 days continuously for detection of the type of seizures. The Tonic-clonic and Absent type seizures were decided to detect. All the 100 subjects were asked to wear the cap and the innerwear with sensors and video camera along with the accelerometer for two-three hours daily alternately. These hours were decided by the experts based on the history of that respective subjects and other symptoms.

Image processing based face and feature detection: Viola-Jones algorithm gives best results for detection of face and features of the region of interest as shown in Figure.6 and Table 1.

Table 1 Overall accuracy of Face \& Feature
extraction using Viola Jones Algorithm
\begin{tabular}{|c|c|}
\hline Features & Detection Accuracy \\
\hline Face & $90 \%$ \\
\hline Mouth & $80 \%$ \\
\hline Eye & $90 \%$ \\
\hline Eyebrow & $80 \%$ \\
\hline
\end{tabular}

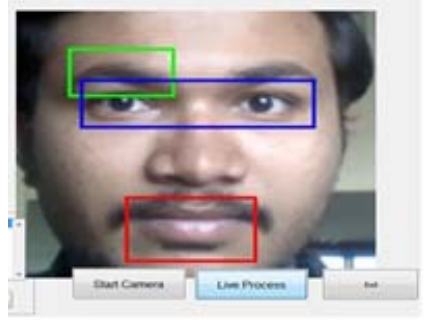

Fig.6. Face and feature detection output

Only 39 subjects have reported facing the seizure during this duration. The details of the experiment are as shown in Table 2

\section{V.CONCLUSION}

The Viola-Jones detection algorithm outcome provides promising results for detection of the face and the region of interest. This performance is supported by the embedded system with wearable sensors. These results describe tonic-clonic seizures and absence seizures. From the results, it may be concluded that this is an on-obtrusive seizure detection environment. In future, a robust decision support system may be undertaken for development. Wearable sensors such as an accelerometer, moisture sensor, body temperature sensors are cost efficient to detect various seizures. So the combination of image processing and embedded system used is novel and efficient for detection and monitoring of different types of epileptic seizures for various patients. 
Table 2 Experimental results

\begin{tabular}{|c|c|c|c|c|c|c|c|}
\hline \multirow{3}{*}{$\begin{array}{l}\text { Sr. } \\
\text { No. }\end{array}$} & \multirow[t]{3}{*}{ Subject No. } & \multicolumn{5}{|c|}{ Symptoms recorded during seizure } & \multirow{3}{*}{$\begin{array}{c}\begin{array}{c}\text { Detection of the Class } \\
\text { of Seizures }\end{array} \\
\text { Tonic-clonic } \\
\text { (A) } \\
\text { Or } \\
\text { Absence } \\
\text { (B) }\end{array}$} \\
\hline & & \multicolumn{3}{|c|}{ Image Processing } & \multicolumn{2}{|c|}{ Wearable Sensor } & \\
\hline & & $\begin{array}{c}\text { Eye } \\
\text { detection }\end{array}$ & $\begin{array}{c}\text { Mouth detection } \\
\text { (Contraction) }\end{array}$ & $\begin{array}{c}\text { Eyebrow } \\
\text { detection } \\
\text { (rigidness) }\end{array}$ & $\begin{array}{c}\text { Accelerometer } \\
\text { (Head movement) }\end{array}$ & $\begin{array}{c}\text { Moisture sensor } \\
\text { (VWC) } \\
\%\end{array}$ & \\
\hline 1 & 3 & $\sqrt{ }$ & $\sqrt{ }$ & $\sqrt{ }$ & Abnormal & 5 & A \\
\hline 2 & 5 & $\sqrt{ }$ & $\sqrt{ }$ & $\sqrt{ }$ & Abnormal & 0 & B \\
\hline 3 & 8 & $\sqrt{ }$ & $\sqrt{ }$ & - & - & 7 & A \\
\hline 4 & 12 & $\sqrt{ }$ & $\sqrt{ }$ & $\sqrt{ }$ & Abnormal & 5 & A \\
\hline 5 & 13 & $\sqrt{ }$ & & $\sqrt{ }$ & Abnormal & 7 & A \\
\hline 6 & 15 & & $\sqrt{ }$ & - & - & 0 & B \\
\hline 7 & 18 & $\sqrt{ }$ & $\sqrt{ }$ & $\sqrt{ }$ & Abnormal & 10 & A \\
\hline 8 & 19 & $\sqrt{ }$ & $\sqrt{ }$ & $\sqrt{ }$ & Abnormal & 5 & $\mathrm{~A}$ \\
\hline 9 & 22 & $\sqrt{ }$ & $\sqrt{ }$ & $\sqrt{ }$ & Abnormal & 7 & A \\
\hline 10 & 25 & $\sqrt{ }$ & $\sqrt{ }$ & - & Abnormal & 5 & $\mathrm{~A}$ \\
\hline 11 & 26 & $\sqrt{ }$ & $\sqrt{ }$ & & Abnormal & 0 & B \\
\hline 12 & 28 & $\sqrt{ }$ & $\sqrt{ }$ & $\sqrt{ }$ & - & 5 & $\mathrm{~A}$ \\
\hline 13 & 29 & & $\sqrt{ }$ & $\sqrt{ }$ & Abnormal & 7 & $\mathrm{~A}$ \\
\hline 14 & 31 & $\sqrt{ }$ & & $\sqrt{ }$ & Abnormal & 10 & A \\
\hline 15 & 34 & $\sqrt{ }$ & $\sqrt{ }$ & $\sqrt{ }$ & - & 0 & $\mathrm{~B}$ \\
\hline 16 & 37 & $\sqrt{ }$ & $\sqrt{ }$ & $\sqrt{ }$ & Abnormal & 5 & $\mathrm{~A}$ \\
\hline 17 & 39 & $\sqrt{ }$ & & $\sqrt{ }$ & Abnormal & 7 & $\mathrm{~B}$ \\
\hline 18 & 43 & $\sqrt{ }$ & $\sqrt{ }$ & $\sqrt{ }$ & - & 0 & $\mathrm{~B}$ \\
\hline 19 & 45 & $\sqrt{ }$ & & $\sqrt{ }$ & Abnormal & 7 & A \\
\hline 20 & 47 & $\sqrt{ }$ & $\sqrt{ }$ & $\sqrt{ }$ & Abnormal & 5 & $\mathrm{~A}$ \\
\hline 21 & 50 & $\sqrt{ }$ & $\sqrt{ }$ & $\sqrt{ }$ & Abnormal & 0 & $\mathrm{~B}$ \\
\hline 22 & 53 & $\sqrt{ }$ & & $\sqrt{ }$ & Abnormal & 0 & $\mathrm{~B}$ \\
\hline 23 & 56 & $\sqrt{ }$ & $\sqrt{ }$ & $\sqrt{ }$ & Abnormal & 5 & $\bar{A}$ \\
\hline 24 & 58 & $\sqrt{ }$ & $\sqrt{ }$ & $\sqrt{ }$ & Abnormal & 10 & $\mathrm{~A}$ \\
\hline 25 & 60 & & $\sqrt{ }$ & $\sqrt{ }$ & - & 0 & $\mathrm{~B}$ \\
\hline 26 & 62 & $\sqrt{ }$ & $\sqrt{ }$ & $\sqrt{ }$ & Abnormal & 5 & $\mathrm{~A}$ \\
\hline 27 & 64 & $\sqrt{ }$ & $\sqrt{ }$ & $\sqrt{ }$ & Abnormal & 7 & A \\
\hline 29 & 65 & $\sqrt{ }$ & $\sqrt{ }$ & 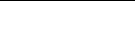 & Abnormal & 0 & B \\
\hline 30 & 66 & $\sqrt{ }$ & $\sqrt{ }$ & $\sqrt{ }$ & - & 10 & $\mathrm{~A}$ \\
\hline 31 & 69 & $\sqrt{ }$ & $\sqrt{ }$ & $\sqrt{ }$ & Abnormal & 5 & $\mathrm{~A}$ \\
\hline 32 & 71 & $\sqrt{ }$ & $\sqrt{ }$ & $\sqrt{ }$ & Abnormal & 7 & $\mathrm{~A}$ \\
\hline 33 & 74 & $\sqrt{ }$ & $\sqrt{ }$ & $\sqrt{ }$ & Abnormal & 0 & $\mathrm{~B}$ \\
\hline 34 & 75 & $\sqrt{ }$ & $\sqrt{ }$ & $\sqrt{ }$ & - & 10 & $\mathrm{~A}$ \\
\hline 36 & 77 & $\sqrt{ }$ & $\sqrt{ }$ & $\sqrt{ }$ & Abnormal & 5 & A \\
\hline
\end{tabular}




\begin{tabular}{|c|c|c|c|c|c|c|c|}
\hline 37 & 79 & $\sqrt{ }$ & $\sqrt{ }$ & $\sqrt{ }$ & Abnormal & 10 & A \\
\hline 38 & 83 & $\sqrt{ }$ & $\sqrt{ }$ & $\sqrt{ }$ & - & 5 & A \\
\hline 39 & 84 & $\sqrt{ }$ & $\sqrt{ }$ & $\sqrt{ }$ & Abnormal & 0 & B \\
\hline
\end{tabular}

\section{REFERENCE}

[1]. Iasem idis LD. ,"Seizure prediction and its applications.", Neuro surg Clin N Am;22:489-506,2011.

[2]. Kwan P, Brodie MJ. ,"Early identification of refractory epilepsy", N Engl J Med;342:314-9,2000.

[3]. Camfield P, Camfield C. ,"Idiopathic generalized epilepsy with generalized tonic- clonic seizures (IGEGTC): a population-based cohort with N20 year follow up for medical and social outcome." Epilepsy Behavior; 18:61,2010.

[4]. Webber WR, Lesser RP, Richardson RT, Wilson K. ,"An approach to seizure detection using an artificial neural network (ANN).", Electroencephalography Clinical Neurophysiology;98:250-72,1996.

[5]. Gabor A J.," Seizure detection using a self-organizing neural network: validation and comparison with other detection strategies.", Electroencephalography Clinical Neurophysiology;107:27-32,1998.

[6]. Wilson SB, Scheuer ML, Emerson RG, Gabor AJ., "Seizure detection: evaluation of the Reveal algorithm.", Clinical Neurophysiology;115:2280-91,2004.

[7]. Pradhan N, Sadasivan PK, Arunodaya GR.,"Detection of seizure activity in EEG by an artificial neural network: a preliminary study.", Computerized Biomedical Resource;29:303-13, 1996

[8]. Petersen EB, Duun-Henriksen J,Mazzaretto A, Kjaer TW, Thomsen CE, Sorensen HB., "Generic singlechannel detection of absence seizures.", Conf Proc IEEE Eng Med Biol Soc ,48203,2011.

[9]. Chan AM, Sun FT, Boto EH,Wingeier BM., "Automated seizure onset detection for accurate onset time determination in intracranial EEG.", Clin Neurophysiol;119:2687-96,2008.

[10]. Conradsen I, Beniczky S, Wolf P, Henriksen J, Sams T, Sorensen HB., "Seizure onset detection based on a uni-or multi-modal intelligent seizure acquisition (UISA/ MISA) system. ",Conf Proc IEEE Eng Med Biol Soc ;:3269-72,2010.

[11]. Jansen K, Lagae L.,"Cardiac changes in epilepsy. Seizure",;19:455-60,2010.

[12]. Leutmezer F, Schernthaner C, Lurger S, Potzelberger K, Baumgartner C., "Electrocardiographic changes at the onset of epileptic seizures. ", Epilepsia;44:348-54,2003.

[13]. Opherk C, Coromilas J, Hirsch LJ. ,"Heart rate and EKG changes in 102 seizures: analysis of influencing factors. ",Epilepsy Res;52:117-27,2002.

[14]. Nijsen TM, Arends JB, Griep PA, Cluitmans PJ. ,"The potential value of three dimensional accelerometry for detection of motor seizures in severe epilepsy.", Epilepsy Behav;7:74-84,2005.

[15]. Lockman J, Fisher RS, Olson DM.,"Detection of seizure-like movements using a wrist accelerometer", Epilepsy Behav;20:638-41,2011.

[16]. Pediaditis M, Tsiknakis M, Leitgeb N., "Vision-based motion detection, analysis and recognition of epileptic seizures—a systematic review.", Computer Methods Programs Biomed;108:1133-48,2012.

[17]. Li Z, Martins da Silva A, Cunha JP., "Movement quantification in epileptic seizures: a new approach to video-EEG analysis.", IEEE Trans Biomed Eng;49:565-73, 2002

[18]. Shoeb A, Pang T, Guttag J, Schachter S., "Non-invasive computerized system for automatically initiating vagus nerve stimulation following patient-specific detection of seizures or epileptiform discharges.", Int J Neural Syst;19:157-72, 2009

[19]. Poh MZ, Loddenkemper T, Reinsberger C, Swenson NC, Goyal S, Sabtala MC, et al. ,"Convulsive seizure detection using a wrist-worn electrodermal activity and accelerometry biosensor. ",Epilepsia;53:e93-7. 2012

[20]. Y. Tian, T. Kanade, J.F. Cohn, "Facial Expression Analysis in Handbook of Face Recognition", Springer, 2005.

[21]. P. Ekman and W. Friesen, "Facial Action Coding System: A Technique for the Measurement of Facial Movement". Consulting Psychologists Press, Palo Alto, 1978.

[22]. M. Pediaditis, M. Tsiknakis, N. Leitgeb, Vision-based human motion detection, analysis and recognition in epilepsy a systematic review, Comput. Meth. Prog. Bio., submitted for publication, 2011.

[23]. Li, Z, da Silva, AM, Cunha, JPS. "Movement quantification in epileptic seizures: a new approach to video-EEG analysis". IEEE Trans Biomed Eng.,49(6):565 573. 2002

[24]. C. Papageorgiou, M. Oren, and T. Poggio. ,"A general framework for object detection", In International Conference on Computer Vision, 1998. 
[25]. Paul Viola, Michael Jones," Rapid Object Detection using a Boosted Cascade of Simple Features", Conference on Computer Vision and Pattern Recognition 2001, 